\title{
Variable Body Biasing (VBB) based VLSI Design Approach to Reduce Static Power
}

\author{
Woo Wei Kai ${ }^{1}$, Nabihah binti Ahmad ${ }^{2}$, Mohamad Hairol bin Jabbar ${ }^{3}$ \\ ${ }^{1,2}$ Department of Electronic Engineering, Universiti Tun Hussein Onn Malaysia (UTHM), Malaysia \\ ${ }^{3}$ Department of Computer Engineering, Universiti Tun Hussein Onn Malaysia (UTHM), Malaysia
}

\section{Article Info \\ Article history: \\ Keyword: \\ 1-Bit full adder \\ Low power \\ Static power \\ VBB technique \\ VLSI design}

Received Jun 122017

Revised Jun 28, 2017

Accepted Aug 26, 2017

\begin{abstract}
The static power consumption is an important parameter concern in IC design due to $\mathrm{t}$ for a higher integration numbers of transistor to achieve greater performance in a single chip. Leakage current is the main issues for static power dissipation in standby mode as the size of transistor been scale. Therefore, the subthreshold leakage current rises due to threshold voltage scaling and gate leakage current increases due to scale down of oxide thickness. In this paper, a Variable Body Biasing (VBB) technique was applied to reduce static power consumption in VLSI design. The VBB technique used a DC bias at body terminal to control the threshold voltage efficiently. The Synopsys Custom Designer EDA tools in 90nm MOSFET technology was used to design a 1-bit full adder with VBB technique in full custom methodology. The simulation of 1-bit full adder was carried out with operation voltage $V_{D D}=0.2 \mathrm{~V}$ supply was compared in conventional technique and VBB technique. The results achieved the reduction in term of peak power, $P_{\text {peak }}=33 \%$ and average power, $P_{\text {avg }}=13 \%$ in static CMOS 1-bit full adder compared with conventional bias and VBB technique.
\end{abstract}

Copyright $\odot 2017$ Institute of Advanced Engineering and Science. All rights reserved.

\section{Corresponding Author:}

Nabihah binti Ahmad,

Department of Electronic Engineering,

Faculty of Electrical and Electronic Engineering (FKEE),

Universiti Tun Hussein Onn Malaysia (UTHM),

Parit Raja 86400 Batu Pahat Johor Malaysia.

Email: nabihah@uthm.edu.my

\section{INTRODUCTION}

In nowadays technology, the size of transistors was scaling down to sub-nanometer (nm) to achieve higher density number of transistor to design a complex, high performance circuit in a smaller size of area. The scaling down in Metal Oxide Semiconductor Field Effect Transistor (MOSFET) channel size is a big challenge for the Integrated Circuit (IC) designers as it doubles every two years according to Moore's law [1].

As the MOSFET technology channel size downscaling, the subthreshold leakage current exponentially increases as threshold voltage $\left(\mathrm{V}_{\mathrm{th}}\right)$ reduces [2]. In a sub-nanometer of transistor, the shorter channel length of transistor will be resulting sub-threshold leakage current through at transistor when in standby mode. Low threshold voltage also results in increased sub-threshold leakage current because transistors cannot be turned OFF completely. Therefore, the static power consumption will occur due to the leakage current when the transistor in standby mode. As a result, it will consume leakage current dissipation, in which has become a significant portion of total power consumption for future IC design technologies in a smaller MOSFET channel length.

In a shorter channel length between Source and Drain of a MOSFET, it will down scaled the transistor threshold voltage $\left(\mathrm{V}_{\mathrm{TH}}\right)$ to maintain a reasonable gate over drive [2]. The MOSFET $\mathrm{V}_{\mathrm{TH}}$ reduction, 
result in an exponential increases in the subthreshold current. Moreover, to control the short channel effects (SCEs) and to maintain the transistor drive strength at low supply voltage, the oxide thickness needs to be also scaled down. The aggressive scaling of oxide thickness results in a high tunneling current through the transistor gate insulator.

In MOSFET, the subthreshold conduction takes place when the applied gate voltage is under the threshold voltage $\left(\mathrm{V}_{\text {th }}\right)$. In long channel devices the $\mathrm{V}_{\mathrm{TH}}$ is independent of the Drain bias, but in sub-nm channel length devices the scenario is different and causes Drain Induced Barrier Lowering (DIBL). The general equation for subthreshold leakage is [2]:

$$
I_{\text {sub }}=I_{0} e^{\left(V_{G S}-V_{T}\right) / n V_{T H}}
$$

where $I_{0}=\mu_{0} c_{o x} \frac{W}{L}(n-1) \mathrm{V}_{\mathrm{TH}}{ }^{2}, V_{G S}$ is the gate-source voltage, $V_{T}$ is the thermal voltage $(K T / q), n$ is the subthreshold swing coefficient, $V_{\text {th }}$ is the threshold voltage, $\mu_{0}$ is the carrier mobility at zero bias, $c_{o x}$ is the gate oxide capacitance, $W$ and $L$ are the effective transistor width and length.

To minimize the subthreshold leakage current dissipation, this paper proposed a Variable Body Bias (VBB) technique to reduce static power consumption in VLSI design. However, there are several VLSI design techniques to reduce leakage power nowadays. Each technique provides an efficient way to reduce leakage power, but the drawback of each technique limit the application of each technique.

\section{RESEARCH METHOD ON VBB TECHNIQUE}

Typically, the body terminal from a MOSFET is used to control the threshold voltage, $V_{\text {th }}$. This benefit made convenient to the IC designers in controlling the $V_{\text {th }}$ by biasing the body of MOSFET independently. This independent body bias dynamically varies the threshold voltage of the MOSFET and the effect of $\mathrm{V}_{\mathrm{TH}}$ variation due to body bias is known as body effect. The basic equation which shows how body bias impacts on threshold voltage $V_{\text {th }}$ is [3]:

$$
\mathrm{V}_{\mathrm{th}}=\mathrm{V}_{\mathrm{th} 0}+\gamma\left(\sqrt{2 \emptyset_{B}-\mathrm{V}_{\mathrm{SB}}}-\sqrt{2 \emptyset_{B}}\right)
$$

where $V_{\text {th }}$ is the threshold voltage, the $\emptyset_{B}$ is the flatband voltage, $\gamma$ is the body effect coefficient, $V_{\text {tho }}$ is the threshold voltage with zero substrate bias and $\mathrm{V}_{\mathrm{SB}}$ is the source to body bias voltage.

According to Equation (2), $\mathrm{V}_{\mathrm{TH} 0}, \gamma$ and $\varnothing$ are the constant element at which setting by the technology's parameter. Therefore, based on Equation (2) the threshold voltage, $V_{\text {th }}$ is directly proportional with the source to body bias voltage, $\mathrm{V}_{\mathrm{SB}},\left(\mathrm{V}_{\mathrm{th}} \propto \mathrm{V}_{\mathrm{SB}}\right)$. Therefore, the body terminal of a MOSFET able to control the threshold voltage, $\mathrm{V}_{\text {th }}$.

Conventionally, in order to maintain the minimum threshold voltage $\left(\mathrm{V}_{\mathrm{th}}\right)$ in static CMOS configuration, the body terminals of pMOS is connected to $V_{D D}$ and the nMOS body terminals connected to ground. In the proposed VBB technique, the logic the body of the pMOS connected to a positive body biased $\left(+V_{B B}\right)$ to increase their $V_{t h}$ in standby mode. Meanwhile, the nMOS body was connected in negative body biased $\left(-\mathrm{V}_{\mathrm{BB}}\right)$ to increase the $\mathrm{V}_{t h}$ because of the body-bias effect in static mode. Figure 1 illustrates the conventional static CMOS connection and VBB technique connection logic design [4].

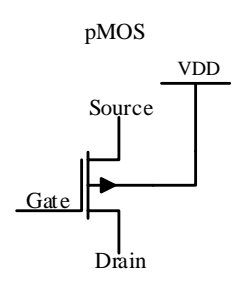

(a)

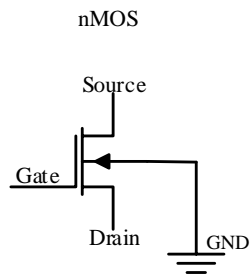

(b)

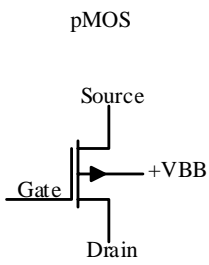

(c)

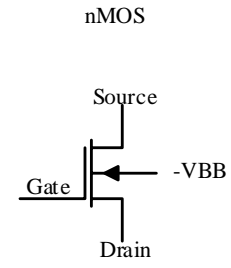

(d)

Figure 1. Body connection of logic design (a), (b) in conventional, (c) and (d) in VBB technique.

The proposed circuit design using VBB technique dynamically control the threshold voltage of transistors through body biasing in standby mode. The body biasing in pMOS biases the body of the transistor to a voltage higher than $\mathrm{V}_{\mathrm{DD}}$, while in nMOS to a voltage lower than $\mathrm{V}_{\mathrm{SS}}$ thereby reducing leakage 
current. To be more understanding of VBB technique, a schematics of CMOS inverter with VBB technique circuit was carried out. Figure 2 illustrates an inverter design with VBB technique.

Based on Figure 2, there was an external supply connected to the body of each MOSFET. Since raising threshold voltage will affects performance and the VBB technique control the body to be applied different input voltage supply, so during an active mode of operation the reverse bias is small, while in standby the reverse bias is stronger. Therefore, it can be reducing the sub-threshold leakage current and reduction of static power dissipation [5].

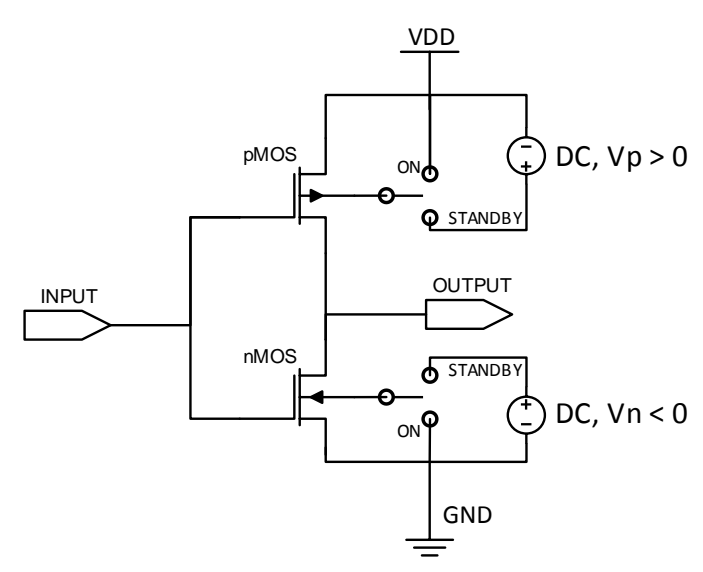

Figure 2. An inverter with VBB technique

\section{VBB TECHNIQUE IN 1-BIT FULL ADDER}

In this paper, the 1-bit full adder was designed in conventional bias and VBB technique. The results obtained were compared and analyze in term of power consumption, propagation delay and number of transistor designed in 1-bit full adder. A 1-bit full adder is a combinational circuit which results in arithmetic sum of two bits. Typically, a full adder has three input terminals and two output terminal. The three inputs are denoted by A, B and a carry input, $\mathrm{C}_{\mathrm{in}}$ and produce two output namely as summation, SUM and carry out, $\mathrm{C}_{\text {out }}$. The truth table of a full adder is shown in Table 1.

Table 1. Truth table of a 1-bit full adder

\begin{tabular}{ccccc}
\hline & \multicolumn{3}{c}{ INPUT } & \multicolumn{2}{c}{ OUTPUT } \\
\hline A & B & Cin & SUM & Cout \\
0 & 0 & 0 & 0 & 0 \\
0 & 0 & 1 & 1 & 0 \\
0 & 1 & 0 & 1 & 0 \\
0 & 1 & 1 & 0 & 1 \\
1 & 0 & 0 & 1 & 0 \\
1 & 0 & 1 & 0 & 1 \\
1 & 1 & 0 & 0 & 1 \\
1 & 1 & 1 & 1 & 1 \\
\hline
\end{tabular}

From the truth table of full adder, the Boolean equation for SUM and $\mathrm{C}_{\text {out }}$ can be obtain by solving with Karnaugh map. The equation for full adder is shown as below:

$$
\begin{aligned}
\mathrm{SUM} & =\mathrm{A} \overline{\mathrm{B}} \overline{\mathrm{C}}+\overline{\mathrm{A}} \mathrm{B} \overline{\mathrm{C}}+\overline{\mathrm{A}} \overline{\mathrm{B}} \mathrm{C}+\mathrm{ABC} \\
& =(\mathrm{A} \oplus \mathrm{B}) \oplus \mathrm{C} \\
\mathrm{C}_{\text {out }} & =\mathrm{AB}+\mathrm{AC}+\mathrm{BC} \\
& =\mathrm{AB}+\mathrm{C}(\mathrm{A}+\mathrm{B}) \\
& =\overline{(\overline{\mathrm{A}}+\mathrm{B})(\overline{\mathrm{C}}+\overline{\mathrm{A}} \overline{\mathrm{B}})} \\
& =\overline{\overline{\mathrm{A}} \overline{\mathrm{B}}+\overline{\mathrm{C}}(\overline{\mathrm{A}}+\overline{\mathrm{B}})}
\end{aligned}
$$


Nowadays, the number of transistors had been reduced to achieve low power consumption in a system. Conventionally, the static CMOS full adder design at which the number of pMOS pull-up network (PUN) and nMOS pull-down network (PDN) must be equivalent. Based on Equation (4) and Equation (5), the static CMOS 1-bit full adder can be design in 28T (Transistor) based on the following equation which is obtained by rearranging the SUM equation and factoring SUM in order to reuse the $\mathrm{C}_{\text {out }}$ term as below:

$$
\mathrm{SUM}=\mathrm{ABC}+(\mathrm{A}+\mathrm{B}+\mathrm{C}) \mathrm{C}_{\text {out }}
$$

Rather than following the conduct complements rule, the pMOS PUN in the adder circuit is identical to the nMOS network according Equation (6). The area efficiency can be obtained in layouts due to this simplification which provides uniformity CMOS design by reducing the number of transistors connected in series. Thus, the static CMOS 28T 1-bit full adder was become the most common full adder in IC design. The schematics of the static CMOS 1- bit full adder using 28T [7] shown in Figure 3.
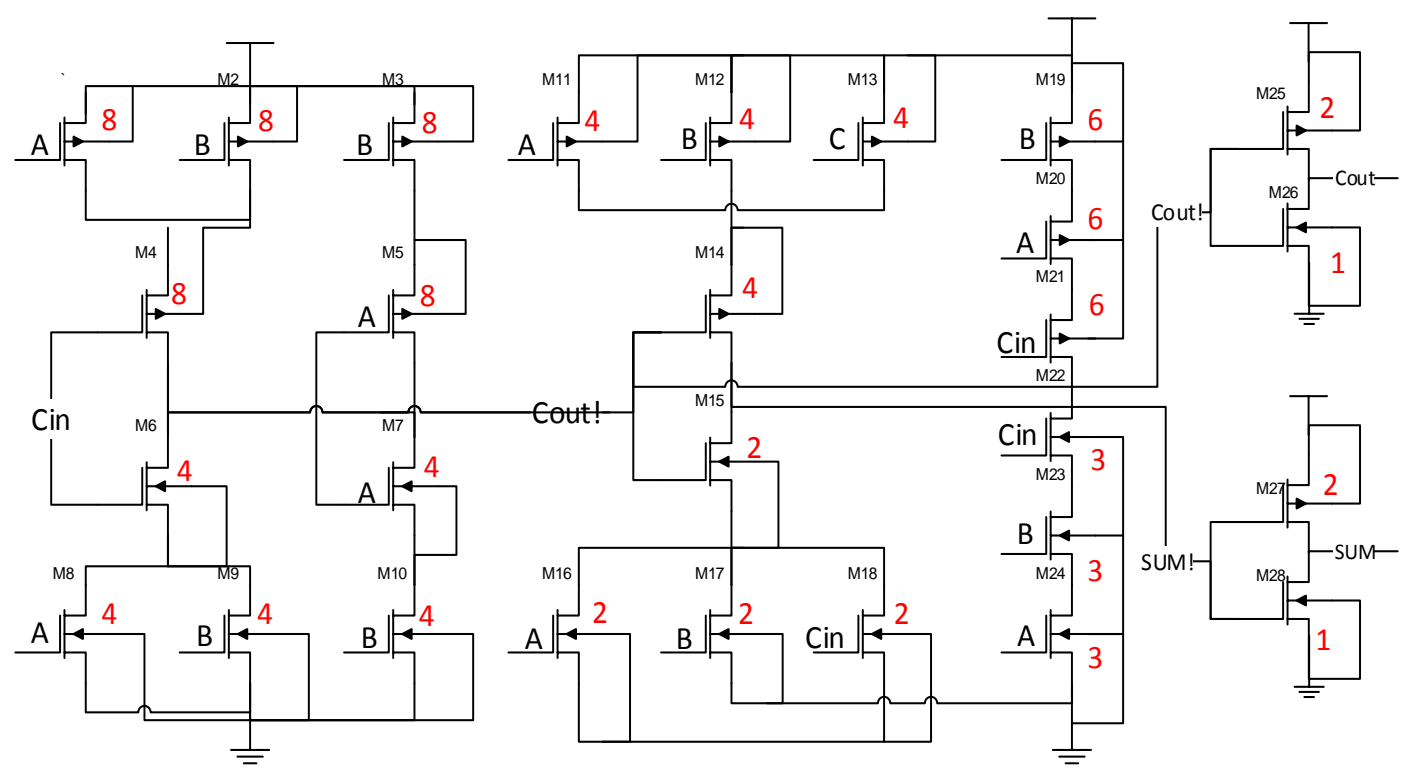

Figure 3. A static CMOS 1-bit full adder with 28T

The MOSFET in designed the 1-bit full adder size was in term of $\left(\frac{W}{L}\right)$ ratio were carried out based on the logical effort calculation in order to optimize the propagation delay. The logical effort of a gate is defined as the ratio of the input capacitance of the gate to the input capacitance of an inverter that can deliver the same output current. Equivalently logical effort indicates how much worse a gate is at producing output current as compared to an inverter. Table 2 shows the logical effort of basic common gate that will be used for design 1-bit full adder [6].

Table 2. Logical effort of basic common gate

\begin{tabular}{ccccccc}
\hline Gate type & \multicolumn{6}{c}{ Number of inputs } \\
Inverter & 1 & 2 & 3 & 4 & 5 & $\mathrm{n}$ \\
NAND & 1 & $4 / 3$ & $5 / 3$ & $6 / 3$ & $7 / 3$ & $(\mathrm{n}+2) / 3$ \\
NOR & & $5 / 3$ & $7 / 3$ & $9 / 3$ & $11 / 3$ & $(2 \mathrm{n}+1) / 3$ \\
\hline
\end{tabular}

Conventionally, the full adder of $28 \mathrm{~T}$, the pMOS is connected to $\mathrm{V}_{\mathrm{DD}}$ and nMOS connected to ground used to prevent latch-up in CMOS where the body-source and body-drain diodes should not be forward biased. In the static 28T 1-bit full adder design shown in Figure 3, the logical effort was being calculate in achieve a minimum propagation delay. The sizing in $\left(\frac{\mathrm{W}}{\mathrm{L}}\right)$ ratio of transistors were illustrated in red color number. 
This paper proposed a VBB technique in a conventional static CMOS 28T full adder design, the body of pMOS and nMOS will be connected to an external source supply in order to provide a reverse bias in standby mode to reduce the sub-threshold leakage current. Figure 4 illustrates the schematic of CMOS 28T full adder with VBB technique.

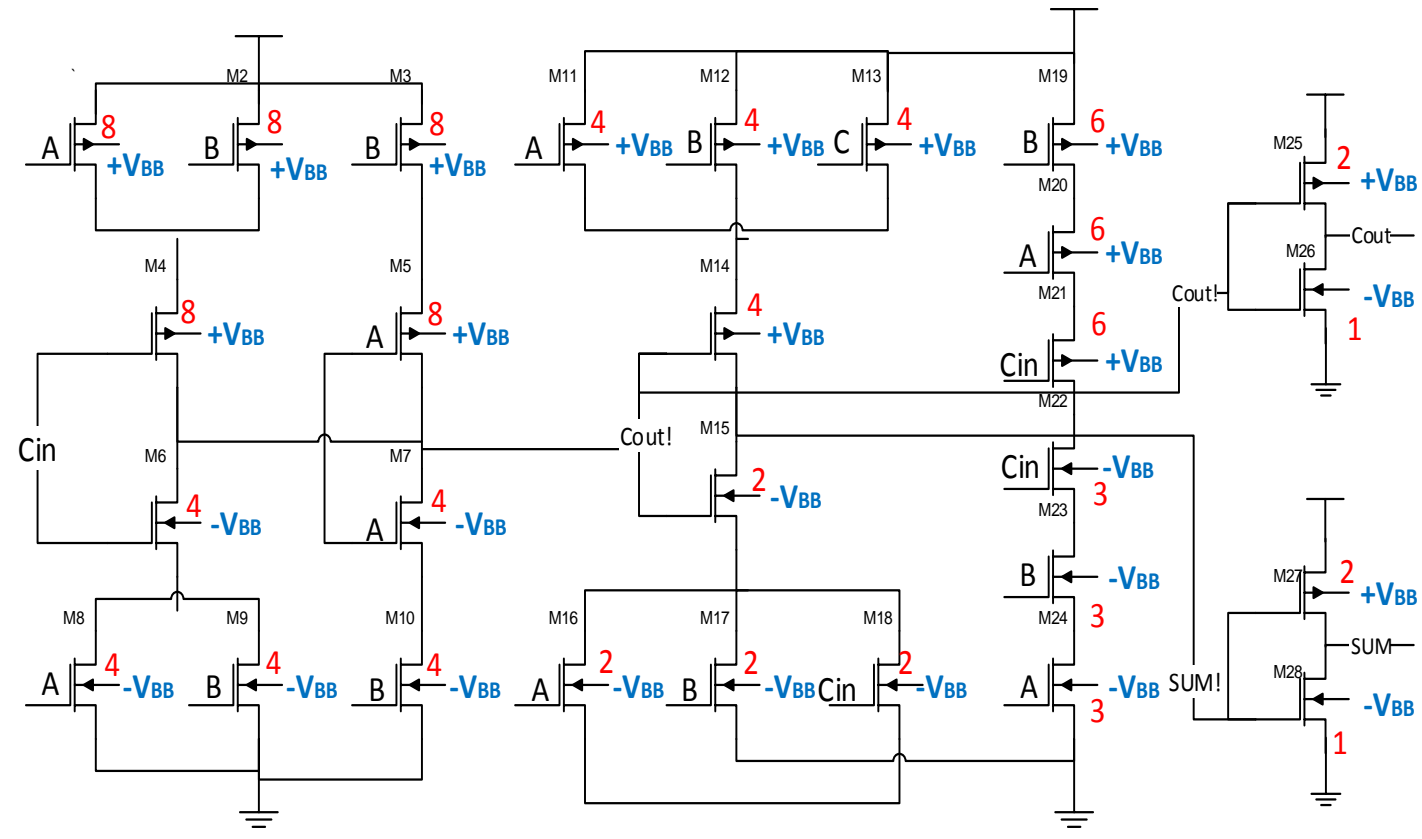

Figure 4. A 28T static CMOS 1-bit full adder with VBB technique

The comparison had been carrier out to analyze performance parameter of power consumption, delay and Power Delay Product (PDP). In order to further reduce the static power consumption, the reduction number of transistor must be done. By resolving and manipulated the Boolean equation of full adder, the number of transistor to design a 1-bit full adder can be reducing by implementation using Complementary Pass Logic transistor (CPL) and Transmission gate design.

The 1-bit full adder circuit with minimize numbers of transistor was being design in 20T [8], 16T [9], 14T [10], 11T [11] and 10T [11] in conventional bias mode and VBB technique to analyze the power consumption. Figure 5 illustrates the 20T 1-bit full adder in transmission gate. Figure 6 illustrates full adder with 16T design in minimize the XOR logic with transmission gate. Figure 7 14T 1-bit full adder was design in CPL with transmission gate. Circuit in Figure 8 and Figure 9 used 11T and 10T 1-bit full adder design with CPL transistor in low power terminology design. The each 1-bit full adder logic style can be used depending upon specific design requirements and constraints imposed by applications. In each of the design the high threshold transistor was been used to reduce the power consumption with high doping concentration of pMOS and nMOS. However, the used of high threshold transistor will increase the propagation delay performance compare with standard MOSFET.

Since this paper focussed on sub-threshold leakage current in which concern on static power dissipation. The design terminology minimizes the used of number transistors designed for 1-bit full adder will not be discussing. The body biasing in conventional and VBB technique of 1-bit full adder was simulating with Synopsis EDA tools of Simulation and Analysis Environment (SAE) in 90nm CMOS technology. The SAE is a comprehensive transistor-level simulation and analysis environment that is tightly integrated and included with Synopsys HSPICE circuit simulators. The netlist-based flow of SAE provides intuitive and comprehensive capabilities to efficiently set up and launch simulations, and analyze and explore simulation results to improve the productivity of analog verification. In order to ensure all the 1-bit full adder running at a similar timing analysis in SAE, the input supply setting was been standardized pulse applied for input $\mathrm{A}, \mathrm{B}$ and $\mathrm{C}_{\text {in }}$ as shown in Table 3 . 


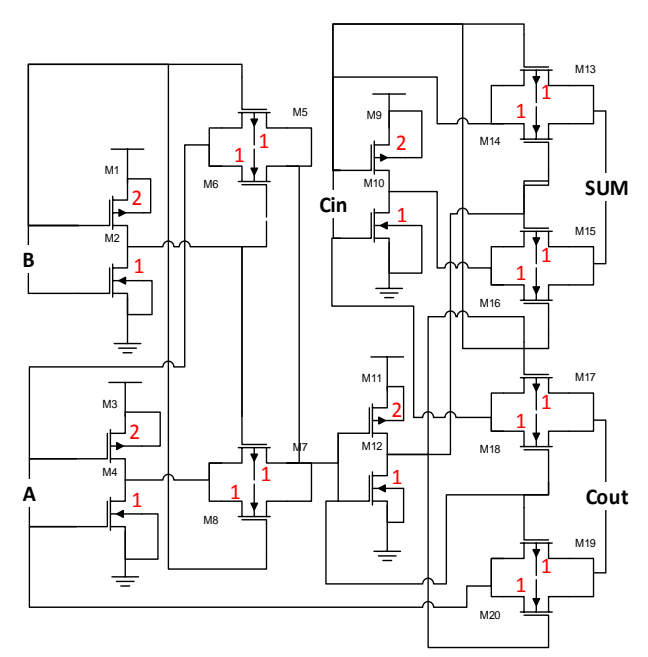

(a)

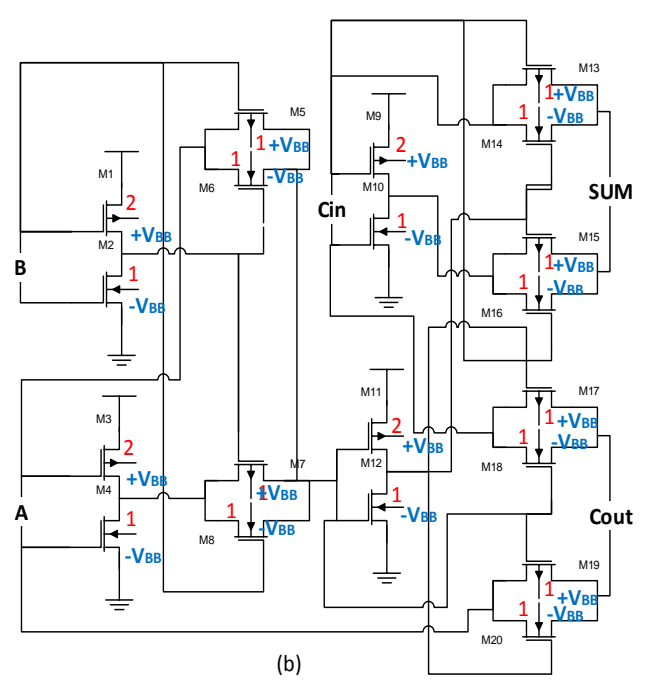

(b)

Figure 5. 20T 1-bit full adder design in (a) conventional bias and (b) VBB technique

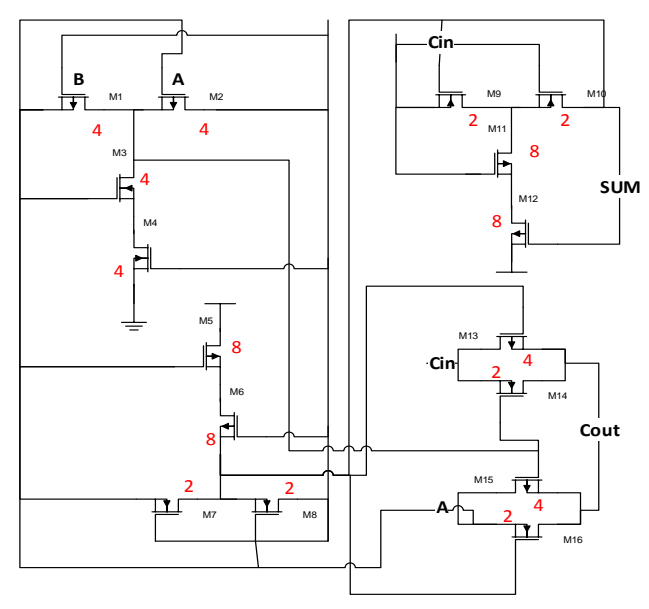

(a)

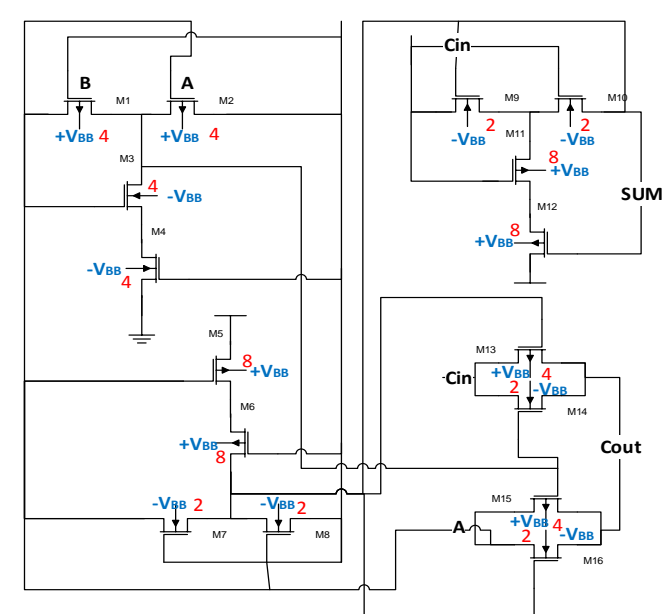

(b)

Figure 6. 16T 1-bit full adder design in (a) conventional bias and (b) VBB technique

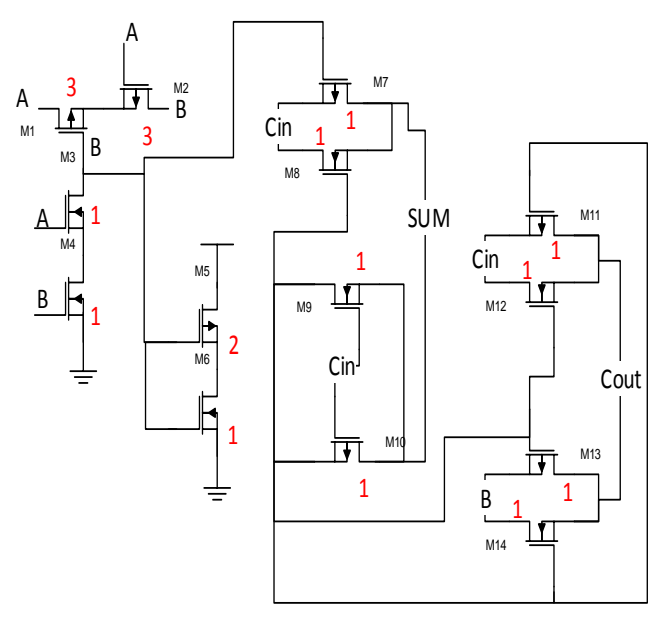

(a)

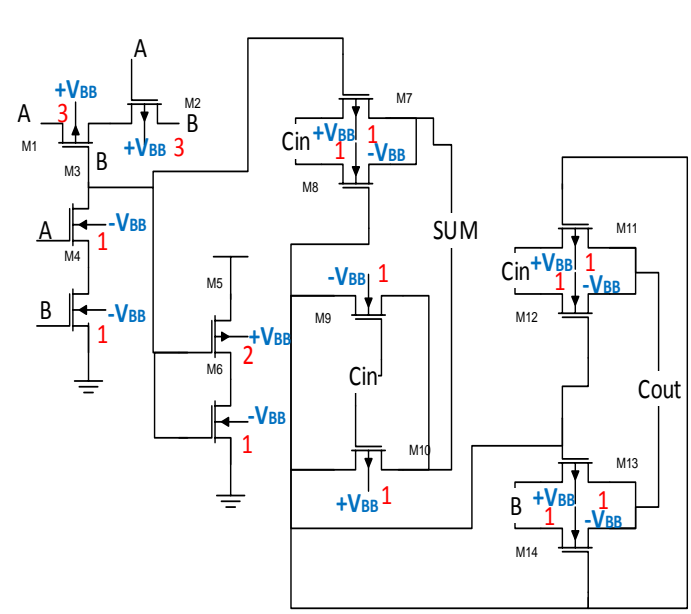

(b)

Figure 7. 14T 1-bit full adder design in (a) conventional bias and (b) VBB technique 


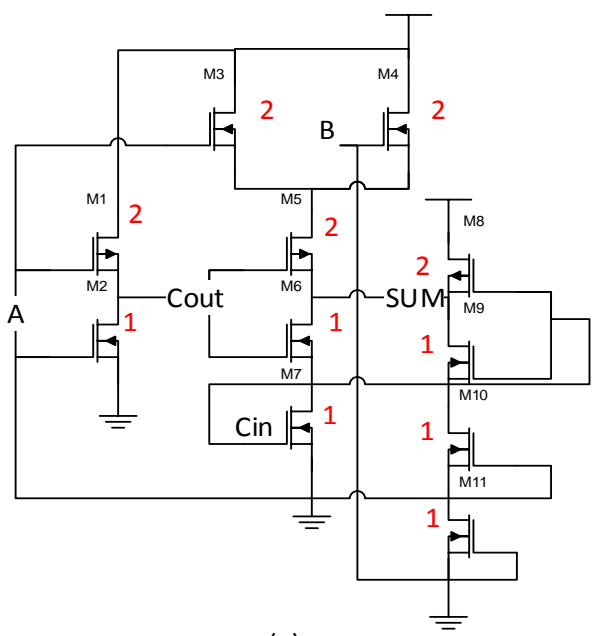

(a)

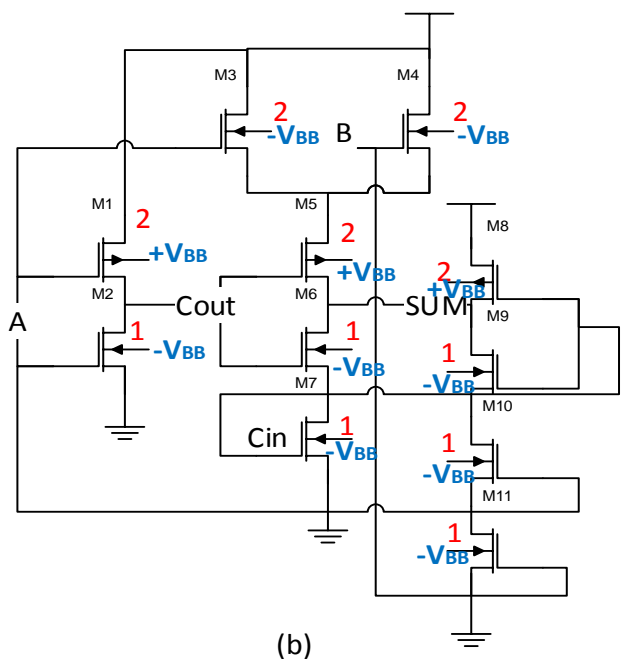

(b)

Figure 8. 11T 1-bit full adder design in (a) conventional bias and (b) VBB technique

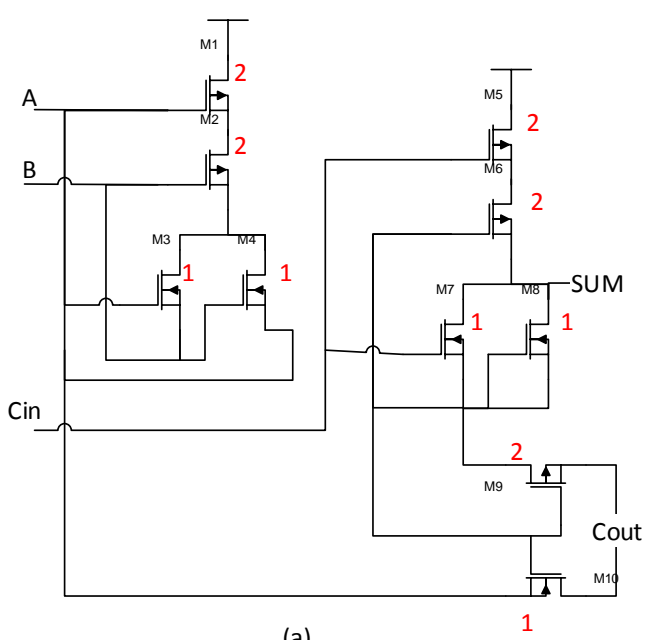

(a)

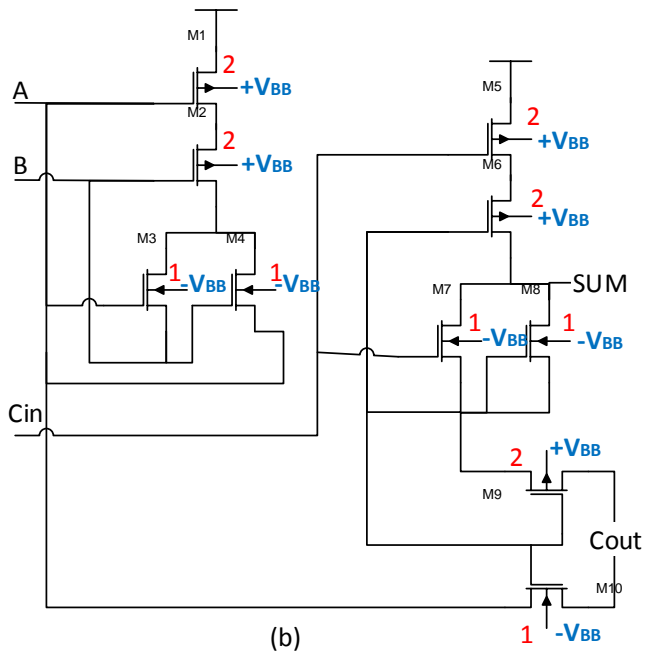

(b)

Figure 9. 10T 1-bit design in (a) conventional bias and (b) VBB technique

Table 3: Standardized input for 1-bit fill adder

\begin{tabular}{cccccc}
\hline INPUT & $\mathrm{V}_{\text {DD }}$ & Rise time & Fall time & Pulse width & Period \\
\hline $\mathrm{B}$ & $0.2 \mathrm{~V}$ & $10 \mathrm{~ns}$ & $10 \mathrm{~ns}$ & $2 \mathrm{us}$ & $4 \mathrm{us}$ \\
$\mathrm{A}$ & $0.2 \mathrm{~V}$ & $10 \mathrm{~ns}$ & $10 \mathrm{~ns}$ & $4 \mathrm{us}$ & $8 \mathrm{us}$ \\
$\mathrm{C}_{\text {in }}$ & $0.2 \mathrm{~V}$ & $10 \mathrm{~ns}$ & $10 \mathrm{~ns}$ & $6 \mathrm{us}$ & $12 \mathrm{us}$ \\
\hline
\end{tabular}

From the inputs pulse setup on Table 3, the most significant bit (MSB) was input $\mathrm{C}_{\text {in }}$ and the least significant bit (LSB) was input B. The pulse width was set with the $50 \%$ of a period. The period of the simulation perform was began at 4 us for input $\mathrm{B}, 8 \mathrm{us}$ for input $\mathrm{A}$ and $12 \mathrm{us}$ for input $\mathrm{C}_{\mathrm{in}}$. Moreover, the rise time and fall time of the pulse width was set at 10ns to observe the glitch intersect occurs on the output.

\section{RESULT AND ANALYSIS}

The 1-bit full adder will be design simulated in Synopsys CMOS 90nm technology tools with the standardize operating voltage of $\mathrm{V}_{\mathrm{DD}}=0.2 \mathrm{~V}$. The functionality of designed 1-bit full adder was verified by comparing with the truth table. 
Furthermore, the comparison of 1-bit full adder continue in term of power performance, propagation delay and power delay product (PDP) were been carried out. Table 4 shown the simulation result of different types of 1-bit full adder with standardize operating voltage of $\mathrm{V}_{\mathrm{DD}}=0.2 \mathrm{~V}$.

Table 4: Simulation result of 1-bit full adder design

\begin{tabular}{|c|c|c|c|c|c|c|}
\hline $\begin{array}{l}\text { Number of } \\
\text { Transistor }\end{array}$ & Body Bias Technique & $\begin{array}{c}\text { Peak Power } \\
(\mathrm{nW})\end{array}$ & $\begin{array}{c}\text { Average Power } \\
\text { (nW) }\end{array}$ & $\begin{array}{c}\text { RMS Power } \\
(\mathrm{nW})\end{array}$ & Delay (ns) & PDP (aJ) \\
\hline \multirow[t]{2}{*}{$28 \mathrm{~T}$} & Conventional & 123.40 & 1.067 & 2.009 & 25.61 & 27.33 \\
\hline & VBB & 82.72 & 0.9275 & 1.447 & 27.43 & 25.44 \\
\hline \multirow[t]{2}{*}{$20 \mathrm{~T}$} & Conventional & 34.25 & 0.6687 & 1.114 & 43.78 & 29.28 \\
\hline & VBB & 30.05 & 0.5913 & 0.8993 & 47.32 & 27.98 \\
\hline \multirow[t]{2}{*}{$16 \mathrm{~T}$} & Conventional & 18.04 & 0.5574 & 0.9152 & 56.76 & 31.64 \\
\hline & VBB & 16.67 & 0.4638 & 0.6787 & 58.92 & 27.33 \\
\hline \multirow[t]{2}{*}{$14 \mathrm{~T}$} & Conventional & 25.23 & 0.3761 & 0.8724 & 57.43 & 21.60 \\
\hline & VBB & 15.38 & 0.2828 & 0.6211 & 59.75 & 16.89 \\
\hline \multirow[t]{2}{*}{$11 \mathrm{~T}$} & Conventional & 13.89 & 0.2043 & 0.5478 & 62.71 & 12.81 \\
\hline & VBB & 13.72 & 0.1321 & 0.4522 & 64.89 & 8.57 \\
\hline \multirow[t]{2}{*}{$10 \mathrm{~T}$} & Conventional & 6.935 & 0.2889 & 0.5486 & 67.34 & 19.45 \\
\hline & VBB & 4.058 & 0.1932 & 0.3004 & 69.28 & 13.38 \\
\hline
\end{tabular}

For the conventional static CMOS 1-bit full adder using 28T, it achieved the reduction power performance in term of $P_{\text {peak }}=33 \%, P_{\text {avg }}=13 \%, P_{R M S}=28 \%$ and PDP reduction of $7 \%$ compared between conventional bias and VBB technique. Therefore, the VBB technique successfully reduce the subthreshold leakage current gradually reduce static power dissipation.

Based on Figure 10 and Figure 11, the reduction of power consumption was gradually reduced as the number transistor in designing 1-bit full adder decreased. Moreover, the proposed VBB technique showed the reduction of static power consumption across the number transistor used. Thus, the minimize usage of transistor was successfully reducing the static power consumption.

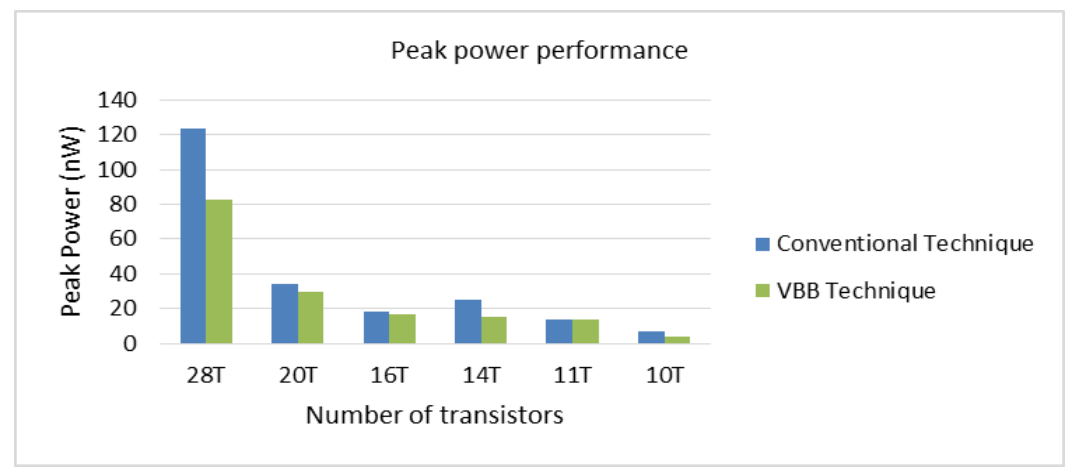

Figure 10. Comparison of peak power performance with number of transistor

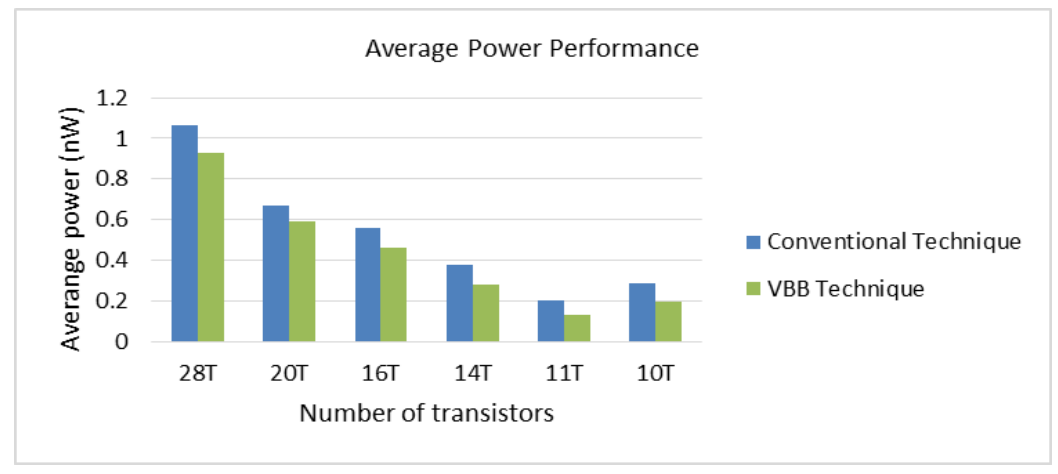

Figure 11. Comparison of average power performance with number of transistor 
Furthermore, the VBB technique have a drawback in the propagation delay at the carry out from the adder summation. Based on Figure 12, there was an increased in delay because of less output driving capability due to the increase in threshold voltage of the device by using VBB technique during standby mode. Moreover, the delay increase because the used of CPL and transmission gate of 1- bit full adder designed. Since the nMOS will pass strong LOW (0) logic while pMOS pass strong HIGH (1) logic, thus in the CPL or transmission gate 1-bit adder designed will be used of an nMOS or pMOS acts as switch to control the flow of input voltage. However, in term of PDP obtained shown that the reducing the numbers of transistor will efficiency reduce the static power consumption.

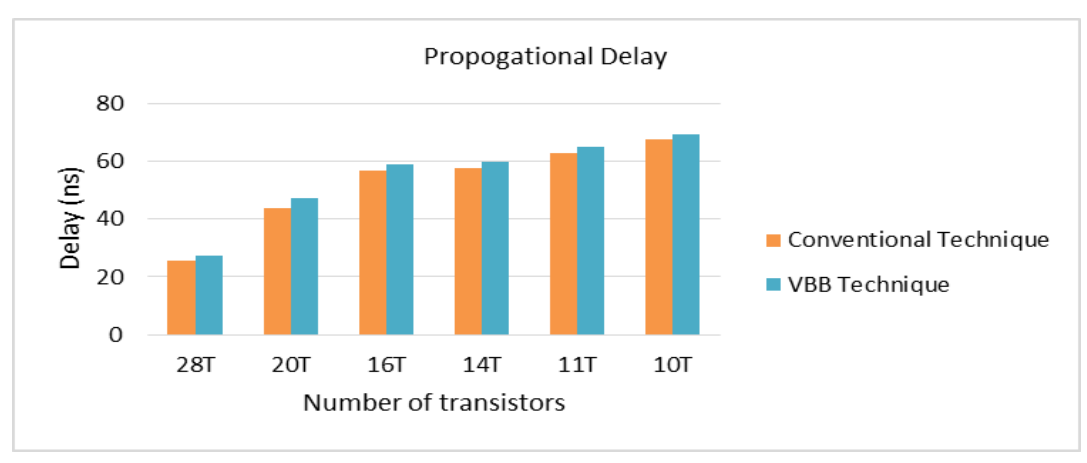

Figure 12. Comparison of propagation delay with no. of transistor

\section{CONCLUSION}

The proposed of Variable Body Bias (VBB) technique in different number of transistors designed in 1 - bit full adder was presented and compared in term of power performance, propagation delay and power delay product (PDP). By comparing in term of static 28T CMOS 1-bit full adder with approached VBB technique was achieved reduction more than $32 \%$ in peak power saving and $13 \%$ in average power but slightly increasing in delay of $7 \%$. Hence from the comparisons, the proposed VBB technique is one of the best alternatives method to achieve low power consumption with accepted performance in VLSI design.

\section{ACKNOWLEDGEMENTS}

This work was financial supported by FRGS Grant Vot Number 1538.

\section{REFERENCES}

[1] Sanapala, Kishore and Sakthivel. R, "Low power realization of subthreshold digital logic circuits using body bias technique", Indian Journal of Science and Technology, 2016, Vol 9(5), pp. 1-5.

[2] R. Gatkal and S. G. Mali, "Low power CMOS inverter in nanometer technology", International Conference on Communication and Signal Processing (ICCSP), 2016, pp. 1982-1986.

[3] Chun, Jae Woong and Chen, Chien-Yi Roger, "Leakage power reduction using the body bias and pin reordering technique", IEICE Electronics Express, 2016, Vol.13(3), pp. 1-7.

[4] A. S. Priyadharshini and A. Veeralakshmi, "Customizable logic cell design using variable body bias", 2nd International Conference on Electronics and Communication Systems (ICECS), 2015, pp. 777-781.

[5] Abhishek Kumar, Effect of Body Biasing Over CMOS Inverter, International Journal of Electronics \& Communication Technology (IJECT), 2013, Vol 4, pp. 369-371.

[6] Paulo Francisco Butzen and Renato Perez Ribas, "Leakage Current in Sub-Micrometer CMOS Gates", Universidade Federal do Rio Grande do Sul, Porto Alegre, Brazil, 2007, pp. 1-30.

[7] Kavita Khare and Krishna Dayal Shukla, "Design A 1-Bit Low Power Full Adder Using Cadence Tool”, MANIT/ Electronics \& Communication, Bhopal, India, 2010, pp. 373-376.

[8] Akansha Maheshwari and Surbhit Luthra, "Low Power Full Adder Circuit Implementation using Transmission Gate", International Journal of Advanced Research in Computer and Communication Engineering, July 2015, Vol. 4, Issue 7, pp. 183-185.

[9] Ebrahim Pakniyat, Seyyed Reza Talebiyan and Milad Jalalian Abbasi Morad, "Design of High performance and Low Power 16T Full Adder Cell for Sub-threshold Technology”, Second International Congress on Technology, Communication and Knowledge (ICTCK), Nov 2015, pp. 79-85. 
[10] Rajesh Parihar, Nidhi Tiwari, Aditya Mandloi and Dr. Binod Kumar, "An Implementation of 1- Bit Low Power Full Adder Based On Multiplexer And Pass Transistor Logic", Information Communication \& Embedded Systems (ICICES), 2014, pp. 1-3.

[11] Hanan A.Mahmoud and Magdy .A. Bayoumi, "A 10-Transistor Low-Power High Speed Full Adder Cell”, The Center for Advance Computer Studies LA, 1999, pp. 43-46.

\section{BIOGRAPHIES OF AUTHORS}
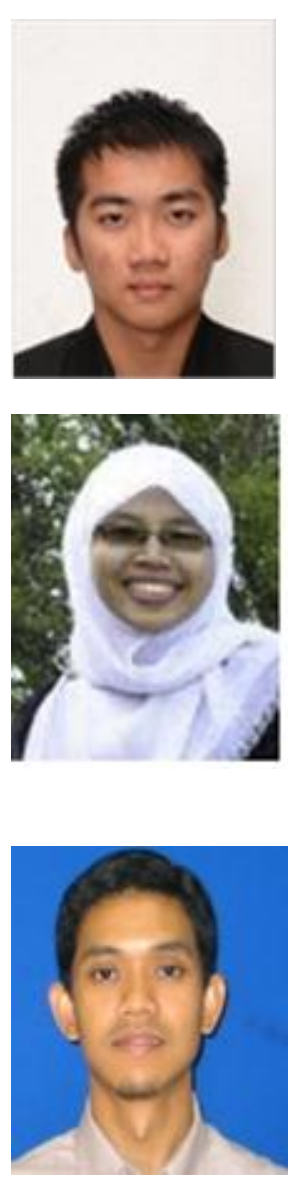

Woo Wei Kai currently an undergraduate student in Electronic Engineering specialized in Microelectonic Engineering from Universiti Tun Hussein Onn Malaysia (UTHM) and received a Diploma in Electrical Enginnering on 2015 in UTHM. Currently he was involving in Integrated Circuit (IC) designed project in term of low power, high performance and smaller in area on $90 \mathrm{~nm}$ technology design. He has done a project such as arithmetric logic unit system, SRAM memory system, variable body bias designed system in full custom methodology. He was familiar in designing circuitry with Synopsys EDA tools at schematics and physical design level of a system.

Nabihah @ Nornabihah bt Ahmad received her Ph.D in Electronic Engineering from Massey University, New Zealand in 2014. She is a senior lecturer at the Department of Electronic Engineering, Faculty of Electrical and Electronic Engineering, Universiti Tun Hussein Onn Malaysia (UTHM). She received a Bachelor of Electrical, Electronic and Systems with Honours from Universiti Kebangsaan Malaysia and Master of Electronic Engineering (Microelectronic) from Kolej Universiti Tun Hussein Onn Malaysia in 2002 and 2005, respectively. She has published a number of research papers in international journals and conferences in the area of Digital, Analog Integrated Circuir Design and VLSI Design. Her research interests include IC design, low power VLSI circuit design, cryptography co-processor, SoC, low power digital system and ASIC/FPGA design. She is currently a member of Board of Engineers Malaysia (BEM), IEEE, IEEE Young Profesionals and IEEE Circuit and System.

Mohamad Hairol Jabbar holds a PhD in Nanotechnology and Nanoelectronic from University of Grenoble, France in 2013. He has published a number of research papers in the past few years. $\mathrm{He}$ is currently working as a lecturer at the Faculty of Electrical and Electronic Engineering, Universiti Tun Hussein Onn Malaysia. His research interests include manycore processor architecture, network on chip, 3D IC, parallel programming and ASIC/FPGA design. 